Walter Eversheim

Organisation in der Produktionstechnik 3 
Springer

Berlin

Heidelberg

New York

Barcelona

Budapest

Hongkong

London

Mailand

Paris

Santa Clara

Singapur

Tokio 
Walter Eversheim

\section{Organisation in der Produktionstechnik 3 \\ Arbeitsvorbereitung}

3., vollständig überarbeitete Auflage

Mit 148 Abbildungen 
Prof. Dr.-Ing. Dr. h.c. Dipl.-Wirt. Ing. Walter Eversheim

RWTH Aachen

WZL Laboratorium für Werkzeugmaschinen

und Betriebslehre

Steinbachstraße 53

52074 Aachen

ISBN-13: 978-3-642-97975-0

Die Deutsche Bibliothek - CIP-Einheitsaufnahme

Eversheim, Walter:

Organisation in der Produktionstechnik / Walter Eversheim. Bd. Arbeitsvorbereitung. - 3., vollst. überarb. Aufl. (VDI-Buch) - Berlin ; Heidelberg ; New York ; Barcelona ; Budapest ; Hongkong; London ; Mailand ; Paris ; Santa Clara ; Singapur ; Tokio : Springer, 1997

Literaturangaben. - Früher im VDI-Verl., Düsseldorf

ISBN-13: 978-3-642-97975-0 e-ISBN-13: 978-3-642-97974-3

DOI: $10.1007 / 978-3-642-97974-3$

Dieses Werk ist urheberrechtlich geschützt. Die dadurch begründeten Rechte, insbesondere die der Übersetzung, des Nachdrucks, des Vortrags, der Entnahme von Abbildungen und Tabellen, der Funksendung, der Mikroverfilmung oder der Vervielfältigung auf anderen Wegen und der Speicherung in Datenverarbeitungsanlagen, bleiben, auch bei nur auszugsweiser Verwertung, vorbehalten. Eine Vervielfältigung dieses Werkes oder von Teilen dieses Werkes ist auch im Einzelfall nur in den Grenzen der gesetzlichen Bestimmungen des Urheberrechtsgesetzes der Bundesrepublik Deutschland vom 9. September 1965 in der jeweils geltenden Fassung zulässig. Sie ist grundsätzlich vergütungspflichtig. Zuwiderhandlungen unterliegen den Strafbestimmungen des Urheberrechtsgesetzes.

(c) Springer-Verlag Berlin, Heidelberg 1997

Softcover reprint of the hardcover 3rd edition 1997

Die Wiedergabe von Gebrauchsnamen, Handelsnamen, Warenbezeichnungen usw. in diesem Werk berechtigt auch ohne besondere Kennzeichnung nicht zu der Annahme, daß solche Namen im Sinne der Warenzeichen- und Markenschutz-Gesetzgebung als frei zu betrachten wären und daher von jedermann benutzt werden dürften.

Sollte in diesem Werk direkt oder indirekt auf Gesetze, Vorschriften oder Richtlinien (z.B. DIN, VDI, VDE) Bezug genommen oder aus ihnen zitiert worden sein, so kann der Verlag keine Gewähr für Richtigkeit, Vollständigkeit oder Aktualität übernehmen. Es empfiehlt sich, gegebenenfalls für die eigenen Arbeiten die vollständigen Vorschriften oder Richtlinien in der jeweils gültigen Fassung hinzuzuziehen.

Einband-Entwurf: Struve \& Partner, Heidelberg

Satz/Datenkonvertierung: MEDIO, Berlin

SPIN: 10568961

89/3020 - Gedruckt auf säurefreiem Papier 


\section{Vorwort zum Kompendium "Organisation in der Produktionstechnik"}

Die Wettbewerbsfähigkeit und die Rentabilität industrieller Unternehmen, insbesondere im Bereich der Investitionsgüterindustrie, wird in entscheidendem Maß durch gezielte Anwendung rationeller Produktionstechniken zur wirtschaftlichen Herstellung von Produkten bestimmt. Vor allem die zunehmende Belastung der Unternehmen durch steigende Personal-, Material- und Energiekosten machen es erforderlich, einerseits das in den Bereichen Konstruktion, Arbeitsvorbereitung, Fertigung und Montage vorhandene technische und organisatorische Potential effektiver zu nutzen und andererseits die Produktionsqualität zu verbessern.

Voraussetzung dafür ist, daß in Konstruktion und Arbeitsvorbereitung moderne Methoden und Hilfsmittel eingesetzt werden, um eine bedarfsgerechte Produktgestaltung und einen zeit- und kostenoptimalen Produktionsablauf zu erzielen.

In der Fertigung und Montage ist hingegen die systematische Planung und Auswahl der geeigneten Produktionsmittel und des einzusetzenden Personals gerade bei angespannter Kapitalsituation von zentraler Bedeutung.

Die vier Bände zum Thema Organisation in der Produktionstechnik wenden sich sowohl an die Studierenden der Fachrichtung Produktionstechnik als auch an alle Fachleute aus der Praxis, die mit organisatorischen Aufgaben in den verschiedenen Produktionsbereichen von Industrieunternehmen beschäftigt sind.

Der Inhalt der vier Bände lehnt sich eng an meine Vorlesung Produktionsmanagement an der Rheinisch-Westfälischen Technischen Hochschule Aachen an und ist wie folgt gegliedert:

Grundlagen,

Konstruktion,

Arbeitsvorbereitung,

Fertigung und Montage. 


\section{Vorwort zu Band 3 „Arbeitsvorbereitung"}

Zunehmende Kapitalintensität und abnehmender Facharbeiteranteil in der Fertigung unterstreichen die Bedeutung der Arbeitsvorbereitung. Aufgrund ihrer Funktion als Bindeglied zwischen Konstruktion und Fertigung kommt der Arbeitsvorbereitung eine besondere Rolle hinsichtlich der Sicherstellung einer wirtschaftlichen Fertigung zu. Darüber hinaus macht der sich in den Betrieben vollziehende technologische Wandel eine ständige Anpassung an sich verändernde Planungsgrundlagen und Planungsaufgaben erforderlich. Dies setzt neben einem breiten Erfahrungsschatz die Kenntnis über bewährte und neue Planungsmethoden, Planungshilfsmittel und Lösungswege voraus.

Dieser Band enthält eine umfassende Darstellung der Aufgaben sowie der anzuwendenden Methoden und Hilfsmittel im Bereich der Arbeitsvorbereitung. Er richtet sich an Studierende des Maschinenbaus und an Praktiker aus dem Bereich der Arbeitsvorbereitung. Aus diesem Grund werden einerseits das erforderliche Grundwissen vermittelt und andererseits die für den Praktiker interessanten Planungshilfsmittel und Planungsmethoden detailliert beschrieben. Darüber hinaus werden die Anbindungspunkte der Arbeitsplanung zur Konstruktion und Fertigung diskutiert und die Funktionalitäten der in der Arbeitsvorbereitung eingesetzten EDV-Hilfsmittel erläutert.

Der Band „Arbeitsvorbereitung“ wurde vollständig überarbeitet und umfaßt die neuesten Forschungsergebnisse und Erkenntnisse im Bereich der Arbeitsvorbereitung. Die gewählte Strukturierung entspricht der systematischen Vorgehensweise in der Arbeitsvorbereitung. Aufgrund ihrer steigenden Bedeutung werden die Themengebiete Arbeitssteuerung sowie Integration in die Unternehmensprozesse in der 3. Neuauflage besonders berücksichtigt.

Der Band entstand unter Mitwirkung meiner Mitarbeiterinnen und Mitarbeiter Dipl.-Ing. St. Breit, Dipl.-Ing. J. Deuse, Dipl.-Ing. A. Haufe, Dipl.-Ing. 
A. Korreck, Dipl.-Ing. G. Kubin, Dipl.-Ing. O. Moron, Dipl.-Ing. D. Much, Dipl.-Ing. Dipl.-Wirt. Ing. M. Munz, Dipl.-Ing. M. Mutz, Dipl.-Ing. P. Ritz, Dipl.-Ing. M. Schotten, Dipl.-Ing. M. Schramm, Dipl.-Ing. I. Schulten, Dipl.Ing. F. Spennemann, Dipl.-Ing. D. Spielberg, Dipl.-Ing. Ch. Vogeler und Dipl.Ing. L. Warnke. Für ihre Einsatzbereitschaft und die jeweiligen Beiträge bedanke ich mich an dieser Stelle ganz herzlich.

Aachen, im April 1997

Walter Eversheim 


\section{Inhalt}

$1 \quad$ Ziele und Gliederung der Arbeitsvorbereitung $\ldots \ldots \ldots \ldots 1$

1.1 Stellung der Arbeitsvorbereitung im Unternehmen ....... 3

1.1.1 Prozeßkette Produktentwicklung ............... 4

1.1.2 Prozeßkette Auftragsabwicklung $\ldots \ldots \ldots \ldots \ldots \ldots \ldots \ldots$

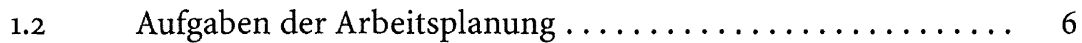

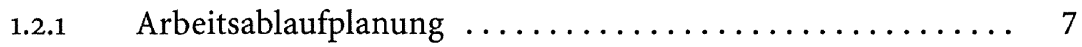

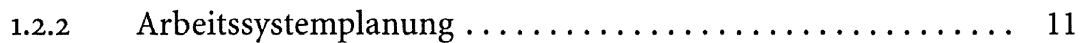

1.3 Aufgaben der Arbeitssteuerung $\ldots \ldots \ldots \ldots \ldots \ldots \ldots$

$2 \quad$ Arbeitsablaufplanung $\ldots \ldots \ldots \ldots \ldots \ldots \ldots \ldots \ldots \ldots$

2.1 Aufgaben der Arbeitsablaufplanung $\ldots \ldots \ldots \ldots \ldots \ldots \ldots$

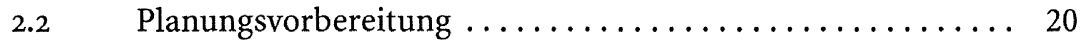

2.3 Stücklistenverarbeitung $\ldots \ldots \ldots \ldots \ldots \ldots \ldots \ldots \ldots \ldots 21$

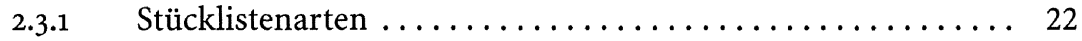

2.3.2 Stücklistenverarbeitung in der Arbeitsvorbereitung ..... 23

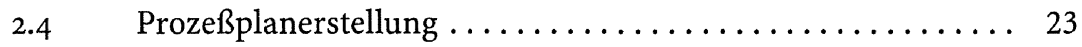

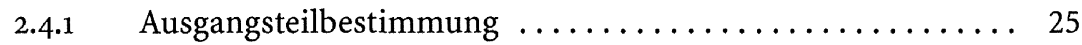

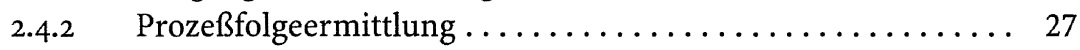

2.4.3 Fertigungsmittelauswahl $\ldots \ldots \ldots \ldots \ldots \ldots \ldots \ldots \ldots \ldots \ldots . \ldots \ldots$

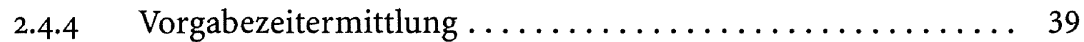

2.4.5 Informationswesen in der Prozeßplanung $\ldots \ldots \ldots \ldots \ldots 43$

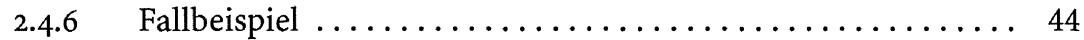

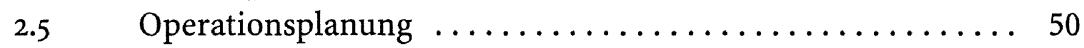

$2.6 \quad$ Montageplanung $\ldots \ldots \ldots \ldots \ldots \ldots \ldots \ldots \ldots \ldots \ldots \ldots \ldots$

2.6.1 Ausprägungen der Montageablaufplanung .......... 57

2.6.2 Hilfsmittel und Methoden zur Montageablaufplanung ..... 60

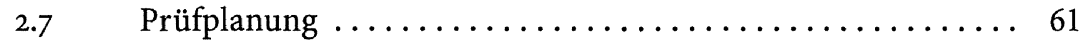

2.7.1 Zielsetzung und Arten der Prüfplanung $\ldots \ldots \ldots \ldots \ldots 62$ 
2.7.2 Durchführung der Prüfplanung $\ldots \ldots \ldots \ldots \ldots \ldots \ldots \ldots 6$

2.7.3 Dokumente der Prüfplanung $\ldots \ldots \ldots \ldots \ldots \ldots \ldots \ldots \ldots$

$2.8 \quad$ Fertigungs- und Prüfmittelplanung $\ldots \ldots \ldots \ldots \ldots \ldots \ldots 6$

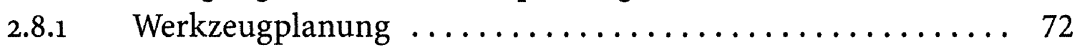

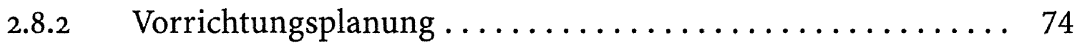

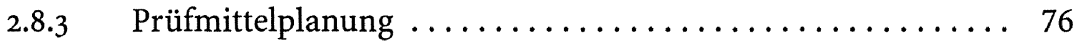

$2.9 \quad$ NC-/ RC-Programmierung $\ldots \ldots \ldots \ldots \ldots \ldots \ldots \ldots \ldots \ldots \ldots$

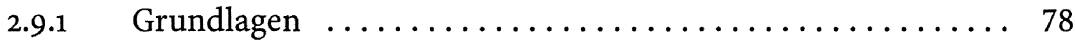

2.9.2 NC-Programmerstellung $\ldots \ldots \ldots \ldots \ldots \ldots \ldots \ldots \ldots \ldots \ldots . \ldots \ldots$

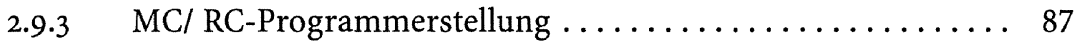

$2.10 \quad$ Kostenplanung/ Kalkulation .................... 89

2.10.1 Ziele, Aufgaben und Einordnung . .............. 89

2.10.2 Kalkulationsverfahren für den wirtschaftlichen $\ldots \ldots \ldots .91$ Verfahrensvergleich

2.10.3 Make-or-buy Entscheidungen ................ 95

$3 \quad$ Arbeitssystemplanung $\ldots \ldots \ldots \ldots \ldots \ldots \ldots \ldots \ldots \ldots$

$3.1 \quad$ Aufgaben der Arbeitssystemplanung $\ldots \ldots \ldots \ldots \ldots \ldots . \ldots 98$

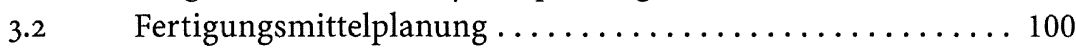

3.2.1 Auswahl der Bearbeitungsmaschinen $\ldots \ldots \ldots \ldots \ldots \ldots 10 \ldots \ldots$

3.2.2 Planung der Anordnungsstruktur . . . . . . . . . . . . . . 104

3.3 Lager- und Transportplanung $\ldots \ldots \ldots \ldots \ldots \ldots \ldots \ldots$

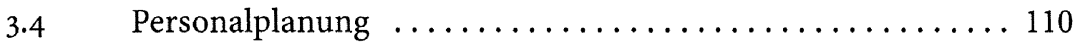

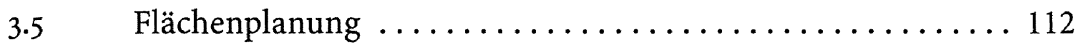

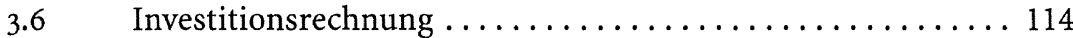

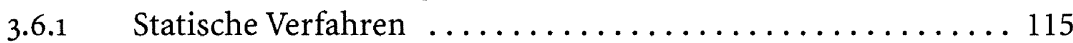

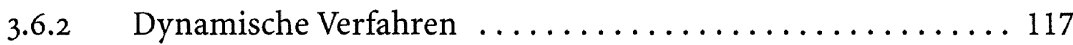

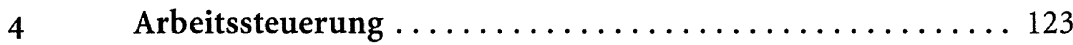

$4.1 \quad$ Aufgaben der Arbeitssteuerung $\ldots \ldots \ldots \ldots \ldots \ldots \ldots \ldots \ldots$

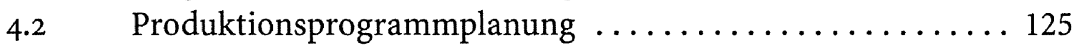

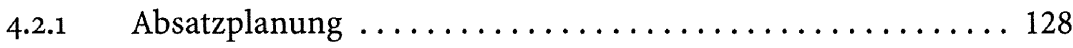

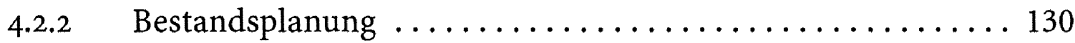

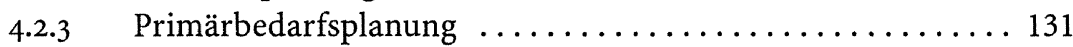

4.2.4 Ressourcengrobplanung (auftragsanonym) $\ldots \ldots \ldots \ldots \ldots 132$

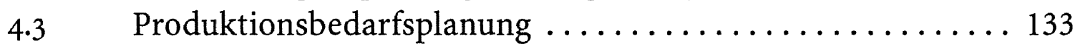

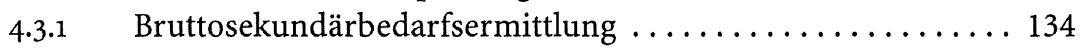

4.3.2 Nettosekundärbedarfsermittlung .............. 136 
4.3.3 Beschaffungsartzuordnung $\ldots \ldots \ldots \ldots \ldots \ldots \ldots \ldots \ldots \ldots \ldots$

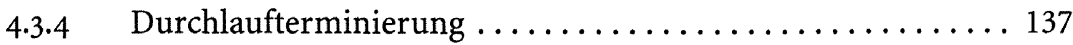

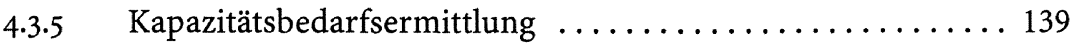

4.3.6 Kapazitätsabstimmung . ................. 139

4.4 Eigenfertigungsplanung und -steuerung $\ldots \ldots \ldots \ldots \ldots \ldots 140$

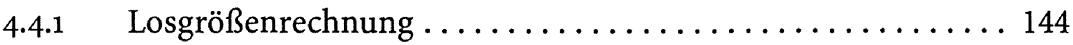

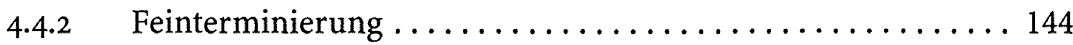

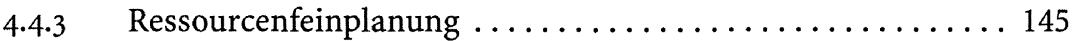

4.4 .4 Reihenfolgeplanung $\ldots \ldots \ldots \ldots \ldots \ldots \ldots \ldots \ldots \ldots \ldots \ldots \ldots$

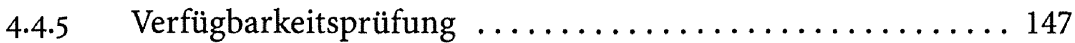

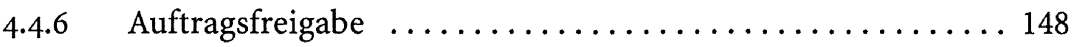

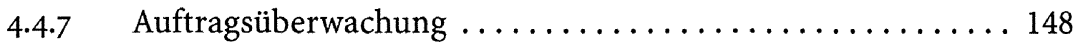

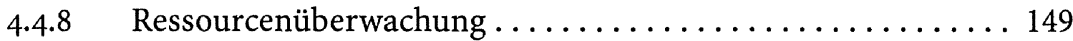

$4.5 \quad$ Fremdbezugsplanung und - steuerung $\ldots \ldots \ldots \ldots \ldots \ldots 149$

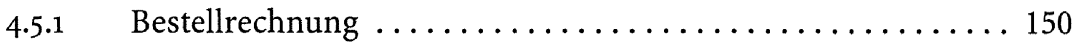

4.5.2 Angebotseinholung/-bewertung $\ldots \ldots \ldots \ldots \ldots \ldots \ldots \ldots 151$

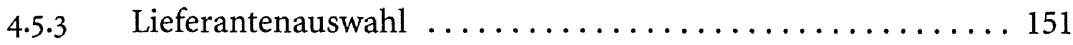

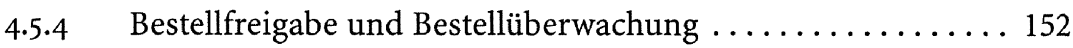

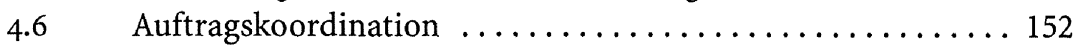

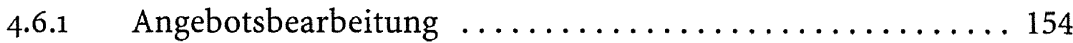

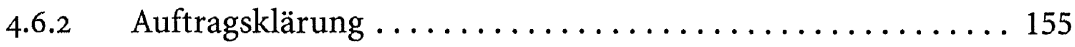

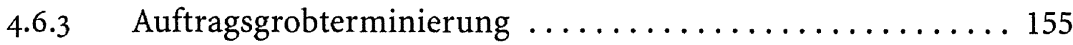

4.6.4 Ressourcengrobplanung (auftragsbezogen) .......... 156

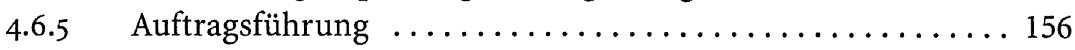

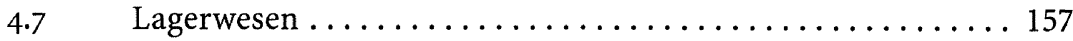

$4.7 .1 \quad$ Lagerbewegungsführung $\ldots \ldots \ldots \ldots \ldots \ldots \ldots \ldots \ldots \ldots \ldots \ldots$

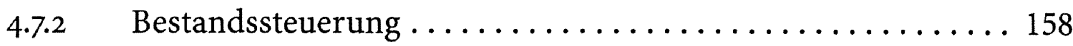

4.7 .3 Lagerort- und Lagerplatzverwaltung . . . . . . . . . . . 159

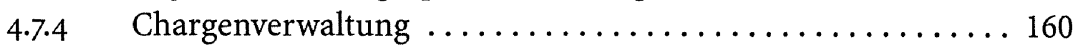

4.7 .5 Lagerkontrolle $\ldots \ldots \ldots \ldots \ldots \ldots \ldots \ldots \ldots \ldots \ldots \ldots \ldots \ldots \ldots$

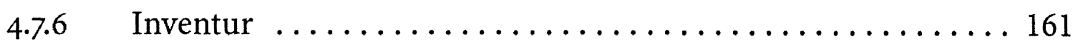

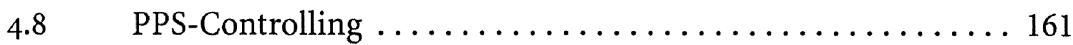

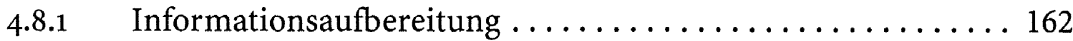

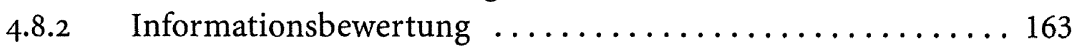

4.8.3 Konfiguration ........................ 164

4.9 Ausprägungen der Arbeitssteuerung . . . . . . . . . 165

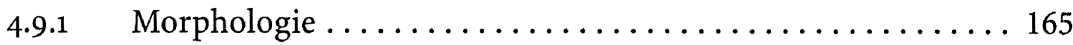

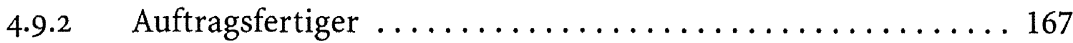


4.9.3 Rahmenauftragsfertiger $\ldots \ldots \ldots \ldots \ldots \ldots \ldots \ldots \ldots \ldots . \ldots \ldots$

4.9.4 Variantenfertiger ............................. 179

4.9.5 Lagerfertiger $\ldots \ldots \ldots \ldots \ldots \ldots \ldots \ldots \ldots \ldots \ldots \ldots \ldots \ldots$

4.10 Ausgewählte Strategien und Verfahren im Rahmen $\ldots \ldots \ldots 187$ der Produktionsplanung und -steuerung

4.10.1 Übersicht . ............................. 188

4.10.2 Management Resources Planning . ................ 190

$4.10 .3 \quad$ Kanban . .................................. 191

4.10.4 Fortschrittszahlenkonzept $\ldots \ldots \ldots \ldots \ldots \ldots \ldots \ldots \ldots \ldots$

4.10.5 Belastungsorientierte Auftragsfreigabe .............. 194

4.10.6 Optimized Production Technology ................. 195

4.10.7 Einordnung und Bewertung $\ldots \ldots \ldots \ldots \ldots \ldots \ldots \ldots \ldots \ldots \ldots$

$5 \quad$ Integration der Arbeitsplanung $\quad \ldots \ldots \ldots \ldots \ldots \ldots \ldots \ldots$........ 199 in die Unternehmensprozesse

5.1 Integration von Konstruktion und Arbeitsplanung ....... 199

5.1.1 Motivation und Zielsetzung . . . . . . . . . . . . . . . . 200

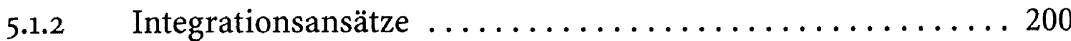

5.2 Integration von Arbeitsplanung und Fertigung $\ldots \ldots \ldots \ldots 204$

5.2.1 Motivation und Zielsetzung . .................... 205

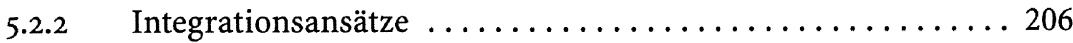

5.2.3 Organisatorische Integration $\ldots \ldots \ldots \ldots \ldots \ldots \ldots \ldots \ldots \ldots \ldots \ldots \ldots$

$6 \quad$ EDV-Systeme in der Arbeitsvorbereitung . . . . . . . . . 213

6.1 Tätigkeitsspezifische EDV-Unterstützung $\ldots \ldots \ldots \ldots \ldots 213$

6.1.1 Rationalisierungsmöglichkeiten durch EDV-Einsatz . . . . . 213 in der Arbeitsplanung

6.1.2 Vorgehensweise zur Rationalisierung $\ldots \ldots \ldots \ldots \ldots \ldots 215$ der Arbeitsplanung

6.1.3 Vorgehensweise zur Einführung von EDV-Systemen ...... 219

6.2 Feature-Technologie ...................... 221

6.2.1 Grundbegriffe der Feature-Technologie ............ 221

6.2.2 Anwendungsfelder der Feature-Technologie ........... 222

6.3 Prozeßplanungssysteme ..................... 224

6.3.1 Funktionalitäten aktueller Prozeßplanungssysteme ....... 225

6.3.2 CAPP-Systeme .......................... 228

6.3.3 Nutzung von Programmierumgebungen $\ldots \ldots \ldots \ldots \ldots 229$ für die Prozeßplanung 


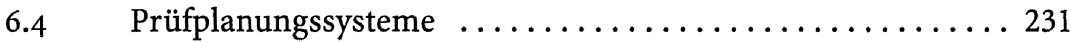

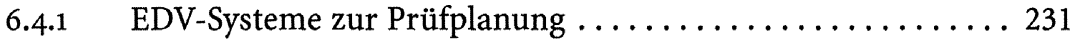

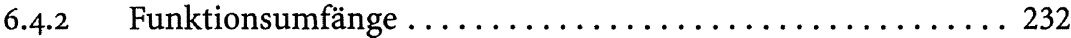

6.4 .3 Schnittstellen zu anderen EDV-Systemen $\ldots \ldots \ldots \ldots \ldots 233$

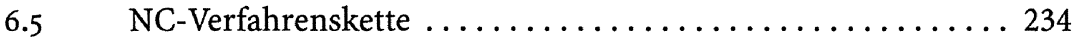

6.5.1 Funktionalitäten aktueller NC-Programmiersysteme . . . . . 237

6.5.2 Verfügbare NC-Programmiersysteme $\ldots \ldots \ldots \ldots \ldots \ldots 241$

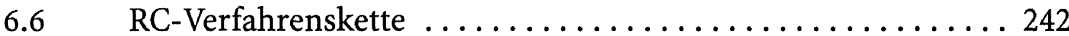

6.6.1 Elemente der RC-Verfahrenskette . . . . . . . . . . . . . . . . 242

6.6.2 Schnittstellen in der NC-Verfahrenskette . . . . . . . . . . 243

6.6.3 Funktionsumfang moderner .................... 244

Off-line-Programmiersysteme

6.6.4 RC-Verfahrenskette am Beispiel Bahnschweißen . . . . . . . . 247

6.7 Betriebsmittelverwaltungssysteme ................ 249

6.7.1 Grundfunktionen von Betriebsmittel- ............. 251 verwaltungssystemen

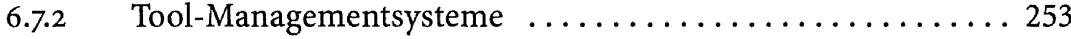

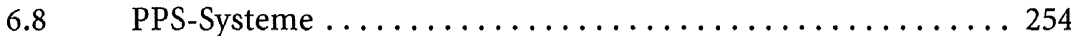

6.8.1 Übersicht . . . . . . . . . . . . . . . . . . . . . . . 255

6.8.2 Systemtechnik von PPS-Systemen $\ldots \ldots \ldots \ldots \ldots \ldots \ldots \ldots 256$

6.8.3 Leistungsumfang von PPS-Systemen ............. 258

6.8.4 Auswahl und Einführung von PPS-Systemen .......... 261

6.9 Integration von EDV-Systemen $\ldots \ldots \ldots \ldots \ldots \ldots \ldots \ldots \ldots \ldots \ldots \ldots \ldots$

6.9.1 Argumente für „Computer Integrated Manufacturing“ . . . . 263

6.9 .2 Realisierung von CIM ................... 266

6.9.3 Integrationsschwerpunkte in der Forschung . . . . . . . 271

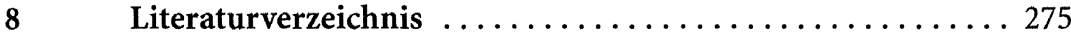

9 Sachwortverzeichnis $\ldots \ldots \ldots \ldots \ldots \ldots \ldots \ldots \ldots \ldots \ldots$ 